\title{
Detecting a Lorentz-Violating Field in Cosmology
}

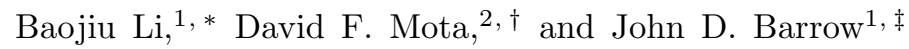 \\ ${ }^{1}$ Department of Applied Mathematics \& Theoretical Physics, \\ Centre for Mathematical Sciences, University of Cambridge, \\ Wilberforce Road, Cambridge CB3 OWA, United Kingdom \\ ${ }^{2}$ Institute of Theoretical Physics, University of Heidelberg, 69120 Heidelberg, Germany
}

(Dated: March 3, 2022)

\begin{abstract}
We consider cosmology in the Einstein-Æther theory (the generally covariant theory of gravitation coupled to a dynamical timelike Lorentz-violating vector field) with a linear Æ-Lagrangian. The $3+1$ spacetime splitting approach is used to derive covariant and gauge invariant perturbation equations which are valid for a general class of Lagrangians. Restricting attention to the parameter space of these theories which is consistent with local gravity experiments, we show that there are tracking behaviors for the $Æ$ field, both in the background cosmology and at linear perturbation level. The primordial power-spectrum of scalar perturbations in this model is shown to be the same that predicted by standard general relativity. However, the power-spectrum of tensor perturbation is different from that in general relativity, but has a smaller amplitude and so cannot be detected at present. We also study the implications for late-time cosmology and find that the evolution of photon and neutrino anisotropic stresses can source the $Æ$ field perturbation during the radiation and matter dominated epochs, and as a result the CMB and matter power spectra are modified. However these effects are degenerate with respect to other cosmological parameters, such as neutrino masses and the bias parameter in the observed galaxy spectrum.
\end{abstract}

PACS numbers: 04.50.+h, 98.80.Jk, 04.80.Cc

\section{INTRODUCTION}

For more than two decades Milgrom's modified Newtonian dynamics (MOND) [1] has been able to explain galaxy rotation curves which are conventionally considered as an evidence of cold dark matter (CDM) on galactic scales. MOND modifies Newton's second law of motion to $\mu\left(|\vec{a}| / a_{0}\right) \vec{a}=-\nabla \Phi_{N}$, where $\vec{a}$ and $\Phi_{N}$ are the acceleration and Newtonian gravitational potential, respectively; $\mu(x)$ is an effectively free function tending to unity in the limit $|\vec{a}| \gg a_{0}$, with $a_{0}$ being a new fundamental constant, which must have a numerical value of $a_{0} \sim(200 \mathrm{~km} / \mathrm{s})^{2} /(10 \mathrm{kpc})$ in order to match observations on a galactic scale. This theory looks like Newton's when accelerations are large but is significantly different when accelerations are small. On galactic scales, $|\vec{a}| \ll a_{0}$, so the Newtonian dynamics is modified, but in a way that can fit spiral galaxy rotation curves. Subsequently, Bekenstein [2] built a relativistic theory which has MOND as a non-relativistic, weak-field limit, thus making the study of cosmology possible. In addition to the conventional tensor gravitational field, Bekenstein's theory involves a vector and a scalar field, and is therefore dubbed TeVeS. Interestingly, it has been argued that TeVeS could also explain the large-scale structure formation of the Universe without recurring to CDM [3], thanks to the presence of the vector field [4].

Recently, the authors of Ref. [5] showed that TeVeS is

\footnotetext{
*Email address: B.Li@damtp.cam.ac.uk

${ }^{\dagger}$ Email address: D.Mota@thphys.uni-heidelberg.de

${ }^{\ddagger}$ Email address: J.D.Barrow@damtp.cam.ac.uk
}

equivalent to a vector-tensor theory of gravitation where the vector field has a non-fixed norm. They also showed that the correct MONDian limit could be realized with a single vector field having non-canonical kinetic terms [6]. These results indicate that a vector field in the gravity sector might be an interesting component of the Universe (indeed the model in [6] and its generalized version we shall presented below in Eq. (11) could be used to explain dark energy and dark matter in background cosmology) and merits more detailed investigations.

The idea of a vector field coupled to gravity has a long worldline (see for example [7] for a review and [8] for further references), but in this work we will focus on the model described in [9], which is the most well-studied one, and investigate its cosmological implications. This particular theory is based on a dynamical vector field coupled to gravitation that picks up a preferred frame and preserves general covariance. This vector field is unitnorm, timelike, and violates local Lorentz invariance. It is called the Æther field (or simply Æ-field) and we will refer to the associated Einstein-Æther theory as Æ-theory defined by the Æ-Lagrangian. The Æ-Lagrangian considered in [9] is a special case of our general model introduced in Eq. (11) below for a unit-norm vector field that includes terms up to second order in derivatives, and it has been extensively studied in various contexts 10, 11, 12, 13, 14].

In Refs. 15, 16], the background cosmology and primordial power spectra of perturbations from inflation of a slight different model were also considered. Here, we investigate these for the model presented in Ref. [9], and also study the evolution of linear perturbation to the $Æ$ field during the radiation and matter-dominated epochs. 
As we will show below, if we restrict the parameter space of the underlying theory so as to satisfy the local experimental gravity constraints, [10, 12], this perturbation becomes sourceless and decays during the epoch of inflation and late-matter domination. However, it is sourced by the evolutions of the photon and neutrino anisotropic stresses during the radiation era and early matter era, which have some imprints on the cosmological observables.

Our presentation is organized as follows. In Sec. II we briefly introduce the general Æ-theory and derive perturbation equations for the background Friedmann-like cosmology in the covariant and gauge invariant (CGI) formalism (see 17] for a derivation of the perturbation equations in conformal Newtonian gauge). In Sec. III we shall use these equations to discuss the perturbation dynamics for the cosmological models of Ref. [9]. First, we summarize the existing constraints on the model in Sec. III A then, in Sec. IIIB, we present the evolution equations for the perturbation variables and then use them to show how the primordial spectra of scalar and tensor perturbations in this theory are unmodified and modified, respectively, on comparing them with the predictions of general relativity (GR). The late-time evolution of the Æ-field perturbation and its effects on cosmological observables are also studied there. Finally, our discussion and conclusions are presented in Sec. [V]

Throughout this work our convention is $\left[\nabla_{a}, \nabla_{b}\right] u^{c}=$ $R_{a b d}^{c} u^{d}, R_{a b}=R_{a c b}^{c}$, where $R_{a b c d}, R_{a b}$ are respectively the Riemann tensor and Ricci tensor; the metric signature is $(+,-,-,-)$ and the universe is assumed to be spatially flat, filled with photons, baryons, CDM, 3 species of neutrinos and a cosmological constant.

\section{FIELD EQUATIONS OF EINSTEIN-ÆTHER THEORY}

In this section we briefly introduce the general form of the $\rightleftarrows$-theory and derive the CGI perturbation equations which we will use to analyze the cosmological effects of the $\rightleftarrows$-field. The equations presented here are for general Lagrangians. Later, we shall focus on a specific class of such theory characterized by a linear Lagrangian (with $f(K)=-K$, see below).

\section{A. The General Einstein- $Æ$ ther Theory}

The model we consider here is a slight generalization of that presented in [6]. It is characterized by a general gravitational action of the form

$$
S=\frac{1}{16 \pi G_{\mathrm{N}}} \int d^{4} x \sqrt{-g}\left[R+\mathcal{L}_{\rightleftarrows}+\lambda\left(\circledast^{a} \circledast_{a}-1\right)\right](1)
$$

in which $G_{\mathrm{N}}$ is the bare Newtonian gravitation constant, $\lambda$ is a Lagrange multiplier ensuring that $Æ$-field has a unit norm, and $\mathcal{L}_{\rightleftarrows}$ is the $Æ$-Lagrangian expressed by

$$
\mathcal{L}_{\rightleftarrows}=f\left(K_{c d}^{a b} \nabla_{a} \boxplus^{c} \nabla_{b} \boxplus^{d}\right)
$$

with

$$
K_{c d}^{a b}=c_{1} g^{a b} g_{c d}+c_{2} \delta_{c}^{a} \delta_{d}^{b}+c_{3} \delta_{d}^{a} \delta_{c}^{b}+c_{4} \mathbb{E}^{a} \mathbb{E}^{b} g_{c d}(3)
$$

and $f(\cdots)$ being an arbitrary analytic function of its arguments. As long as the norm of $\mathbb{E}_{a}$ is fixed $\left(\mathbb{E}^{a} \mathbb{E}_{a}=1\right)$, the form of $K^{a b}$ id the most general possibility we can have for our vector-field Lagrangian. Notice that Eq. (3) here differs from that given in [6] by the $c_{4}$ term and we shall refer to our model and that of [6] as the GEA (Generalized Einstein Æther) model to distinguish from the one considered in [9] (for which we instead called EA (Einstein Æther)). The matter Lagrangian is taken to be the same as in standard $\Lambda \mathrm{CDM}$ model.

We treat the $Æ$-field $\mathbb{E}^{a}$ and inverse metric $g^{a b}$ as the dynamical degrees of freedom and vary the action with respect to them to obtain the field equations. The former gives the $\rightleftarrows$-field equation of motion (EOM):

$$
\nabla_{b}\left(F J_{a}^{b}\right)-c_{4} F Æ^{b} \nabla_{b} Æ^{c} \nabla_{a} Æ_{c}=\lambda Æ_{a}
$$

where we have defined $K \equiv K_{c d}^{a b} \nabla_{a} \mathbb{E}^{c} \nabla_{b} \mathbb{E}^{d}, F \equiv$ $F(K) \equiv \partial f(K) / \partial K$ and $J_{c}^{a} \equiv K_{c d}^{a b} \nabla_{b} \mathbb{E}^{d}$. The variation with respect to the metric leads to a modified Einstein equation. One could retain the form of Einstein equations in standard GR by treating the vector field as a new contribution (denoted by $T_{a b}^{\mathbb{E}}$ ) to the total energymomentum tensor in the universe, in addition to that of the conventional fluid matter which is denoted by $T_{a b}^{f}$. Then, according to the definition

$$
16 \pi G_{\mathrm{N}} T_{a b}^{Æ} \equiv \frac{-2}{\sqrt{-g}} \frac{\delta\left(\sqrt{-g} \mathcal{L}_{\leftarrow}^{\prime}\right)}{\delta g^{a b}}
$$

in which $\mathcal{L}_{\circledast}^{\prime}=\mathcal{L}_{\circledast}+\lambda\left(\circledast^{a} \circledast_{a}-1\right)$, we have

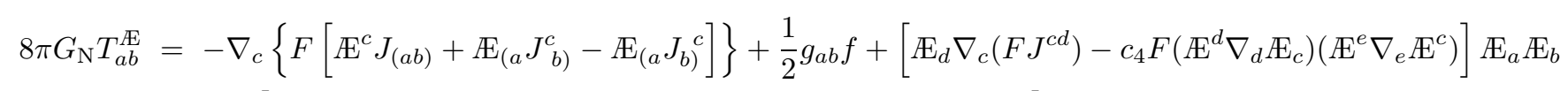

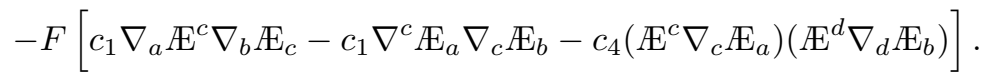


We also note that by varying the action with respect to the Lagrangian multiplier $\lambda$ we simply get the normal-

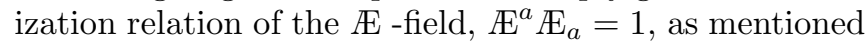
above.

\section{B. The Perturbation Equations in General Relativity}

The CGI perturbation equations in general $\rightleftarrows$-theories are derived in this section using the method of $3+1$ decomposition [18] (see 19] for applications of this method in modified-gravity models). First, we briefly review the main ingredients of $3+1$ decomposition and their application to standard general relativity [18] for ease of later reference.

The main idea of $3+1$ decomposition is to make spacetime splits of physical quantities with respect to the 4velocity $u^{a}$ of an observer. The projection tensor $h_{a b}$ is defined as $h_{a b}=g_{a b}-u_{a} u_{b}$ and can be used to obtain covariant tensors perpendicular to $u$. For example, the covariant spatial derivative $\hat{\nabla}$ of a tensor field $T_{d \cdots e}^{b \cdots c}$ is defined as

$$
\hat{\nabla}^{a} T_{d \cdots e}^{b \cdots c} \equiv h_{i}^{a} h_{j}^{b} \cdots h_{k}^{c} h_{d}^{r} \cdots h_{e}^{s} \nabla^{i} T_{r \cdots s}^{j \cdots k}
$$

The energy-momentum tensor and covariant derivative of the 4-velocity are decomposed respectively as

$$
\begin{aligned}
T_{a b} & =\pi_{a b}+2 q_{(a} u_{b)}+\rho u_{a} u_{b}-p h_{a b}, \\
\nabla_{a} u_{b} & =\sigma_{a b}+\varpi_{a b}+\frac{1}{3} \theta h_{a b}+u_{a} A_{b} .
\end{aligned}
$$

In the above, $\pi_{a b}$ is the projected symmetric trace-free (PSTF) anisotropic stress, $q_{a}$ the heat flux vector, $p$ the isotropic pressure, $\sigma_{a b}$ the PSTF shear tensor, $\varpi_{a b}=$ $\hat{\nabla}_{[a} u_{b]}$ the vorticity, $\theta=\nabla^{c} u_{c} \equiv 3 \dot{a} / a$ ( $a$ is the mean expansion scale factor) the expansion scalar, and $A_{b}=\dot{u}_{b}$ the acceleration; the overdot denotes time derivative expressed as $\dot{\phi}=u^{a} \nabla_{a} \phi$, brackets mean antisymmetrisation, and parentheses symmetrization. The 4-velocity normalization is chosen to be $u^{a} u_{a}=1$. The quantities $\pi_{a b}, q_{a}, \rho, p$ are referred to as dynamical quantities and $\sigma_{a b}, \varpi_{a b}, \theta, A_{a}$ as kinematical quantities. Note that the dynamical quantities can be obtained from the energymomentum tensor $T_{a b}$ through the relations

$$
\begin{aligned}
\rho & =T_{a b} u^{a} u^{b} \\
p & =-\frac{1}{3} h^{a b} T_{a b}, \\
q_{a} & =h_{a}^{d} u^{c} T_{c d} \\
\pi_{a b} & =h_{a}^{c} h_{b}^{d} T_{c d}+p h_{a b} .
\end{aligned}
$$

Decomposing the Riemann tensor and making use the Einstein equations, we obtain, after linearization, five constraint equations [18]:

$$
\begin{aligned}
0 & =\hat{\nabla}^{c}\left(\varepsilon_{c d}^{a b} u^{d} \varpi_{a b}\right) ; \\
\kappa q_{a} & =-\frac{2 \hat{\nabla}_{a} \theta}{3}+\hat{\nabla}^{b} \sigma_{a b}+\hat{\nabla}^{b} \varpi_{a b} ; \\
\mathcal{B}_{a b} & =\left[\hat{\nabla}^{c} \sigma_{d(a}+\hat{\nabla}^{c} \varpi_{d(a}\right] \varepsilon_{b) e c}{ }^{d} u^{e} ; \\
\hat{\nabla}^{b} \mathcal{E}_{a b} & =\frac{1}{2} \kappa\left[\hat{\nabla}^{b} \pi_{a b}+\frac{2}{3} \theta q_{a}+\frac{2}{3} \hat{\nabla}_{a} \rho\right] ; \\
\hat{\nabla}^{b} \mathcal{B}_{a b} & =\frac{1}{2} \kappa\left[\hat{\nabla}_{c} q_{d}+(\rho+p) \varpi_{c d}\right] \varepsilon_{a b}^{c d} u^{b},
\end{aligned}
$$

and five propagation equations,

$$
\begin{aligned}
\dot{\theta}+\frac{1}{3} \theta^{2}-\hat{\nabla}^{a} A_{a}+\frac{\kappa}{2}(\rho+3 p) & =0 ;(15) \\
\dot{\sigma}_{a b}+\frac{2}{3} \theta \sigma_{a b}-\hat{\nabla}{ }_{\langle a} A_{b\rangle}+\mathcal{E}_{a b}+\frac{1}{2} \kappa \pi_{a b} & =0 ;(16) \\
\dot{\varpi}+\frac{2}{3} \theta \varpi-\hat{\nabla}_{[a} A_{b]} & =0 ;(17) \\
\frac{1}{2} \kappa\left[\dot{\pi}_{a b}+\frac{1}{3} \theta \pi_{a b}\right]-\frac{1}{2} \kappa\left[(\rho+p) \sigma_{a b}+\hat{\nabla}_{\langle a} q_{b\rangle}\right] & \\
-\left[\dot{\mathcal{E}}_{a b}+\theta \mathcal{E}_{a b}-\hat{\nabla}^{c} \mathcal{B}_{d(a} \varepsilon_{b) e c}{ }^{d} u^{e}\right] & =0 ;(18) \\
\dot{\mathcal{B}}_{a b}+\theta \mathcal{B}_{a b}+\hat{\nabla}^{c} \mathcal{E}_{d(a} \varepsilon_{b) e c}{ }^{d} u^{e} & \\
+\frac{\kappa}{2} \hat{\nabla}^{c} \pi_{d(a} \varepsilon_{b) e c}{ }^{d} u^{e} & =0 .(19)
\end{aligned}
$$

Here, $\varepsilon_{a b c d}$ is the covariant permutation tensor, $\mathcal{E}_{a b}$ and $\mathcal{B}_{a b}$ are respectively the electric and magnetic parts of the Weyl tensor $\mathcal{W}_{a b c d}$, defined by $\mathcal{E}_{a b}=u^{c} u^{d} \mathcal{W}_{a c b d}$ and $\mathcal{B}_{a b}=-\frac{1}{2} u^{c} u^{d} \varepsilon_{a c}{ }^{e f} \mathcal{W}_{e f b d}$. The angle bracket means taking the trace-free part of a quantity.

Besides the above equations, it is useful to express the projected Ricci scalar $\hat{R}$ into the hypersurfaces orthogonal to $u^{a}$ as

$$
\hat{R} \doteq 2 \kappa \rho-\frac{2}{3} \theta^{2}
$$

The spatial derivative of the projected Ricci scalar, $\eta_{a} \equiv$ $\frac{1}{2} a \hat{\nabla}_{a} \hat{R}$, is then given as

$$
\eta_{a}=\kappa \hat{\nabla}_{a} \rho-\frac{2 a}{3} \theta \hat{\nabla}_{a} \theta
$$

and its propagation equation by

$$
\dot{\eta}_{a}+\frac{2 \theta}{3} \eta_{a}=-\frac{2}{3} \theta a \hat{\nabla} a \hat{\nabla} \cdot A-a \kappa \hat{\nabla}_{a} \hat{\nabla} \cdot q .
$$

Finally, there are the conservation equations for the energy-momentum tensor:

$$
\begin{aligned}
\dot{\rho}+(\rho+p) \theta+\hat{\nabla}^{a} q_{a} & =0 \\
\dot{q}_{a}+\frac{4}{3} \theta q_{a}+(\rho+p) A_{a}-\hat{\nabla}_{a} p+\hat{\nabla}^{b} \pi_{a b} & =0 .
\end{aligned}
$$

As we are considering a spatially-flat universe, the spatial curvature must vanish on large scales and so $\hat{R}=0$. Thus, from Eq. (20), we obtain

$$
\frac{1}{3} \theta^{2}=\kappa \rho \text {. }
$$


This is the Friedmann equation in standard general relativity, and the other background equations (the Raychaudhuri equation and the energy-conservation equation) can be obtained by taking the zero-order parts of Eqs. (15, 23), yielding:

$$
\begin{aligned}
\dot{\theta}+\frac{1}{3} \theta^{2}+\frac{\kappa}{2}(\rho+3 p) & =0 \\
\dot{\rho}+(\rho+p) \theta & =0 .
\end{aligned}
$$

In what follows, we will only consider scalar perturbation modes, for which the vorticity $\varpi_{a b}$ and magnetic part of Weyl tensor $\mathcal{B}_{a b}$ are at most of second order [18], and so will be neglected in our first-order analysis.

\section{The Perturbation Quantities in $Æ$-Theory}

In the Einstein-Æther theories where we consider the $\rightleftarrows$-field as a new species of matter, the gravitational field equations Eqs. (10-27) listed above preserve their forms, but the dynamical quantities $\rho, p, q_{a}, \pi_{a b}$ appearing there should be replaced by the effective total quantities of the same type. For simplicity, we shall always use variables without superscripts to denote these effective total quantities, while for those of a specified matter species we shall add corresponding superscripts (e.g., $\rho^{\circledR}$ denotes the energy density of the $\rightleftarrows$-field $\cdots$..).

The vector $\mathbb{E}$-field, $\mathbb{E}_{a}$, requires further discussion. As we mentioned above, it has the normalization relation $\mathbb{E}^{a} \mathbb{E}_{a}=u^{a} u_{a}=1$. In the background FriedmannRobertson-Walker (FRW) universe the requirements of homogeneity and isotropy require that $\mathbb{E}_{a}$ is just equal to $u_{a}$, which is unambiguously chosen as the 4-velocity of the fundamental observers. But in a perturbed, almostFRW, universe this is no longer true and we can write $\mathbb{E}_{a}=u_{a}+\mathfrak{x}_{a}, \mathbb{E}^{a}=u^{a}+\mathfrak{x}^{a}$ where $\mathfrak{x}_{a}$ is another (firstorder) vector field that vanishes in a FRW Universe: we call it the perturbation of the $\rightleftarrows$-field. Furthermore, the relation $\mathbb{E}^{a} \mathbb{E}_{a}=u^{a} u_{a}=1$ implies that $u^{a} æ_{a}=0$, i.e., $æ_{a}$ is a spatial vector field which is perpendicular to $u_{a}$, up to first order in perturbation. This fact is used extensively in deriving the perturbation equations (e.g., $\nabla^{a} æ_{a}=\hat{\nabla}^{a} æ_{a}$ etc.).

With these preliminaries at hand, and after some lengthy manipulations, the $Æ$-field EOM Eq. (6) can be written as (up to first order)

$$
\begin{aligned}
c_{14}\left[F \ddot{æ}_{a}+(\dot{F}+F \theta) \dot{æ}_{a}\right]+c_{14}\left[F \dot{A}_{a}+\dot{F} A_{a}+\frac{2}{3} F \theta A_{a}\right]-\left[\frac{1}{3}\left(\alpha-c_{14}\right)(\dot{F} \theta+F \dot{\theta})-\frac{2}{9} c_{14} F \theta^{2}\right] æ_{a} \\
+\frac{1}{3} \alpha \hat{\nabla}_{a}(F \theta)+\frac{1}{3} \alpha F \hat{\nabla}_{a} \hat{\nabla}^{b} æ_{b}+c_{13} F \hat{\nabla}^{b}\left(\sigma_{a b}+\hat{\nabla}_{\langle a} æ_{b\rangle}\right)=0
\end{aligned}
$$

and from the definitions Eq. (9) the $\rightleftarrows$-field energy density, isotropic pressure, heat-flux vector and anisotropic stress can be identified from Eq. (7) (again up to first order) as

$$
\begin{aligned}
& \kappa \rho^{\circledR}=\frac{1}{2} f-\frac{1}{3} F \alpha\left(\theta^{2}+2 \theta \hat{\nabla}^{a} æ_{a}\right)+c_{14} F \hat{\nabla}^{a}\left(A_{a}+\dot{æ}_{a}+\frac{1}{3} \theta \mathfrak{x}_{a}\right), \\
& \kappa p^{⿷}=-\frac{1}{2} f+\frac{\alpha}{3} \dot{F}\left(\theta+\hat{\nabla}^{a} æ_{a}\right)+\frac{\alpha}{3} F\left[\dot{\theta}+\theta^{2}+\left(\hat{\nabla}^{a} æ_{a}\right)^{\cdot}+2 \theta \hat{\nabla}^{a} æ_{a}\right], \\
& \kappa q_{a}^{\rightleftarrows}=-c_{14}\left[F \dot{A}_{a}+\dot{F} A_{a}+\frac{2}{3} F \theta A_{a}\right]-c_{14}\left[F \ddot{æ}_{a}+(\dot{F}+F \theta) \dot{æ}_{a}\right]+\left[\frac{1}{3}\left(\alpha-c_{14}\right)(\dot{F} \theta+F \dot{\theta})-\frac{2}{9} c_{14} F \theta^{2}\right] æ_{a} \\
& =\frac{1}{3} \alpha \hat{\nabla}_{a}(F \theta)+\frac{1}{3} \alpha F \hat{\nabla}_{a} \hat{\nabla}^{b} æ_{b}+c_{13} F \hat{\nabla}^{b}\left(\sigma_{a b}+\hat{\nabla}_{\langle a} æ_{b\rangle}\right), \\
& \kappa \pi_{a b}^{Æ}=-c_{13}(\dot{F}+F \theta)\left[\sigma_{a b}+\hat{\nabla}_{\langle a} æ_{b\rangle}\right]-c_{13} F\left[\dot{\sigma}_{a b}+\left(\hat{\nabla}_{\langle a} æ_{b\rangle}\right)^{\cdot}\right],
\end{aligned}
$$

where, in Eq. (32), we have used Eq. (28). Here, we have defined the new parameters $\alpha \equiv c_{1}+3 c_{2}+c_{3}, c_{13} \equiv c_{1}+c_{3}$ and $c_{14} \equiv c_{1}+c_{4} ; \kappa$ is given by $\kappa=8 \pi G_{\mathrm{N}}$. Including these $\rightleftarrows$-contributions to Eqs. (10-27) one obtains the modified gravitational field equations for the general $\rightleftarrows$ -theory. It is also easy to check that the above results satisfy (separately) the conservation of the $\rightleftarrows$-field's energymomentum tensor, Eqs. (23, 24). Note that Eqs. (29]- 
33) are the general expressions of energy density, pressure, heat flux and anisotropic stress in the $3+1$ decomposition which include both zeroth order (background) and first order terms; to calculate the actually density contrast etc. (see Eqs. (51- 54) below) one needs to take the covariant spatial derivatives of these equations [18].

\section{A SPECIFIC MODEL: THE LINEAR LAGRANGIAN}

In above we presented the field equations for general $\rightleftarrows$-theories, but in what follows we shall only analyze the cosmology of a specific edition of the theory which is defined by choice of a linear Lagrangian:

$$
f(K)=-K
$$

(note that the minus sign is because of our sign convention). This model is by far the most well known, in the sense that it has been investigated in the contexts of static weak-field limit [10] (see also [11 for the weak-

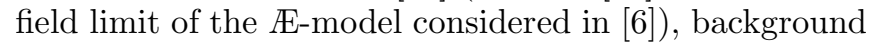
cosmology [15], the radiation and propagation of the $\mathbb{E}$ gravitational waves [12, 16], compact stars [13], and black holes [14]. Some of these studies have imposed stringent constraints on the viable parameter-space of $c_{i}$ s. In view of these restrictions we will confine our study to this constrained subset of possible theories. Note that the perturbation dynamics of the $Æ$-model has also been analyzed in [16] in the absence of the $c_{4}$ term. Here, we shall include this term and perform a similar analysis but in slightly different manner and in more detail; we will also discuss on some detailed features which lead to our conclusions being different. The late-time evolution of the $\mathbb{E}$ -perturbation is also investigated. In particular, we shall find that within the locally-constrained parameter space the $\rightleftarrows$-model will leave slightly different signatures on the perturbation evolutions from those left by the standard $\Lambda \mathrm{CDM}$ paradigm in general relativity, and so cosmological data on cosmic microwave background (CMB) and matter power spectra might place some constraint on the parameter space.

\section{A. The Constrained Parameter Space}

In this subsection we briefly summarize the constraints on the $\rightleftarrows$-model described in Eq. (34). It has been well known that in the weak-field, slow-motion, limit and in the background cosmology the $\rightleftarrows$-model displays tracking behavior. For the former environment, it can be shown that in the presence of the $\mathbb{E}$-field the observed effective gravitational constant $G_{0}$ is a rescaling of the bare one $G_{\mathrm{N}}[10,15]$ by (note that here our $c_{i}$ s have different signs from those in [10])

$$
G_{0}=\frac{1}{1+\frac{1}{2} c_{14}} G_{\mathrm{N}},
$$

and in the latter environment the observed gravitational constant $G_{\infty}$ is also a rescaling of $G_{\mathrm{N}}$, but with a different factor 15 ]

$$
G_{\infty}=\frac{1}{1-\frac{1}{2} \alpha} G_{\mathrm{N}}
$$

Correspondingly, we define $\kappa_{0}=8 \pi G_{0}$ and $\kappa_{\infty}=8 \pi G_{\infty}$ to be used below. Note that the rescaled $G_{\infty}$ is generally not equal to $G_{\mathrm{N}}$ and as such the background cosmic expansion rate will be different from that in standard GR. However, we note that the numerical value of gravitational constant we find in the textbooks and use in the numerical calculations is not $G_{\mathrm{N}}$ but rather the locally-measured $G_{0}$. It is possible to obtain limits on non-local values of $G$ by considering the primordial nucleosynthesis of light elements (see for example Ref. [21]). If $c_{14}=-\alpha$ then we have $G_{\infty}=G_{0}$, which indicates that the background cosmological dynamics will be exactly the same as assuming standard GR [15, 20]; otherwise we can use constraints from primordial nucleosynthesis on the value of gravitational constant to show that $\left|G_{\infty} / G_{0}-1\right| \lesssim \mathcal{O}(0.1)[10]$. However, note that the particle-horizon size at the epoch of neutron-proton freeze-out $(t \sim 1 \mathrm{~s})$, which is most sensitive to variations in the value of $G$, is only $\sim 10^{10} \mathrm{~cm}$ and this causally linked region expands in size by about a factor of $10^{10}$ by the present-day to a size $\sim 10^{20} \mathrm{~cm} \sim 32 \mathrm{pc}$ which is a sub-galactic scale but there has then been local gravitational collapse by a factor of $10^{2}$. Such collapse may however also affect the local value of the gravitational constant 22], but we will not consider this in the present work.

There are also constraints from the observations of parameterized post-Newtonian (PPN) parameters [23]. It is shown in [10] that for all the PPN parameters to coincide with those in GR (otherwise the parameters may need to be fine-tuned) one reduces the full four-parameter space of the model to a two-parameter subspace characterized by

$$
\begin{aligned}
& c_{2}=\frac{-2 c_{1}^{2}-c_{1} c_{3}+c_{3}^{2}}{3 c_{1}}, \\
& c_{4}=-\frac{c_{3}^{2}}{c_{1}} .
\end{aligned}
$$

In addition, $\rightleftarrows$-theories contain five gravitational and $\rightleftarrows$ -wave modes, which include the two usual spin-2 gravitational waves, and three additional modes: two spin-1 transverse $Æ$-gravity waves and one spin-0 longitudinal $\mathbb{E}$-gravity wave. The speeds of the three additional modes are generally not equal to 1 . It has been shown in Ref. [12] that if these speeds are less than 1 then the highenergy particles will produce C̆erenkov radiation when passing through vacuum, which imposes stringent constraints on the model. However, as suggested in [10], these constraints do not apply if these modes propagate superluminally. The requirement that the additional $\rightleftarrows$ - 
gravity waves do not propagate subluminally further limits the parameter space to

$$
-1<c_{13}<0, \quad \frac{c_{13}}{3\left(1+c_{13}\right)}<c_{1}-c_{3}<0 .
$$

Finally, when the above constraints Eq. (38) are satisfied, the positive energy requirement and the stability of additional wave modes [16] also hold. In addition, we will have $c_{14}=-\alpha$ so that the Big Bang nucleosynthesis constraint does not apply. Thus, we can see that, even after using all the current constraints, there is still a large parameter space remaining for the model. In what follows we shall ask whether linear-order cosmological observations such as the CMB and the form of the matter power spectrum could reduce this parameter space further, and as we will show, the answer is positive. However, the modifications are small and depend weakly on the model parameters, which mean that the data on CMB and matter power spectra cannot give very stringent limits on the parameter space.

\section{B. Linearly Perturbed Equations}

In this subsection we consider the perturbation evolu-

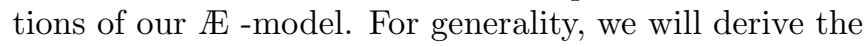
equations for arbitrary choices of parameters and only later confine ourselves to the parameter space described in Eq. (38). Besides, since the presence of the $Æ$-field in general will modify the cosmology at all times, we will also investigate its effects during the inflationary era as in [16]; after that we will turn to its effects on late time cosmology.

Following [18], we shall make the following harmonic expansions of our perturbation variables

$$
\begin{aligned}
& \hat{\nabla}_{a} \rho=\sum_{k} \frac{k}{a} \mathcal{X} Q_{a}^{k}, \quad \hat{\nabla}_{a} p=\sum_{k} \frac{k}{a} \mathcal{X}^{p} Q_{a}^{k} \\
& q_{a}=\sum_{k} q Q_{a}^{k}, \quad \pi_{a b}=\sum_{k} \Pi Q_{a b}^{k}, \\
& \hat{\nabla}_{a} \theta=\sum_{k} \frac{k^{2}}{a^{2}} \mathcal{Z} Q_{a}^{k}, \sigma_{a b}=\sum_{k} \frac{k}{a} \sigma Q_{a b}^{k} \\
& \hat{\nabla}_{a} a=\sum_{k} k h Q_{a}^{k}, \quad A_{a}=\sum_{k} \frac{k}{a} A Q_{a}^{k} \\
& \mathfrak{Q}_{a}=\sum_{k} æ Q_{a}^{k}, \quad \eta_{a}=\sum_{k} \frac{k^{3}}{a^{2}} \eta Q_{a}^{k} \\
& \mathcal{E}_{a b}=-\sum_{k} \frac{k^{2}}{a^{2}} \phi Q_{a b}^{k}
\end{aligned}
$$

in which $Q^{k}$ is the eigenfunction of the comoving spatial Laplacian $a^{2} \hat{\nabla}^{2}$ satisfying

$$
\hat{\nabla}^{2} Q^{k}=\frac{k^{2}}{a^{2}} Q^{k}
$$

and $Q_{a}^{k}, Q_{a b}^{k}$ are given by $Q_{a}^{k}=\frac{a}{k} \hat{\nabla}_{a} Q^{k}, Q_{a b}^{k}=\frac{a}{k} \hat{\nabla}_{\langle a} Q_{b\rangle}^{k}$. Note that $æ$ is dimensionless.

In terms of the above harmonic expansion coefficients, Eqs. (11, 13, 16, 18, 21, 22) can be rewritten as [18]

$$
\begin{aligned}
\frac{2}{3} k^{2}(\sigma-\mathcal{Z}) & =\kappa q a^{2} \\
k^{3} \phi & =-\frac{1}{2} \kappa a^{2}[k(\Pi+\mathcal{X})+3 \mathcal{H} q] \\
k\left(\sigma^{\prime}+\mathcal{H} \sigma\right) & =k^{2}(\phi+A)-\frac{1}{2} \kappa a^{2} \Pi \\
k^{2}\left(\phi^{\prime}+\mathcal{H} \phi\right) & =\frac{1}{2} \kappa a^{2}\left[k(\rho+p) \sigma+k q-\Pi^{\prime}-\mathcal{H} \Pi\right] \\
k^{2} \eta & =\kappa \mathcal{X} a^{2}-2 k \mathcal{H Z} \\
k \eta^{\prime} & =-\kappa q a^{2}-2 k \mathcal{H} A
\end{aligned}
$$

where $\mathcal{H}=a^{\prime} / a=\frac{1}{3} a \theta$ and a prime denotes the derivative with respect to the conformal time $\tau(a d \tau=d t)$. Also, Eq. (24) and the spatial derivative of Eq. (23) become

$$
\begin{aligned}
& q^{\prime}+4 \mathcal{H} q+(\rho+p) k A-k \mathcal{X}^{p}+\frac{2}{3} k \Pi=0 \\
& \mathcal{X}^{\prime}+3 h^{\prime}(\rho+p)+3 \mathcal{H}\left(\mathcal{X}+\mathcal{X}^{p}\right)+k q=0
\end{aligned}
$$

Recall that we shall always neglect the superscript tot for the total dynamical quantities and add appropriate superscripts for individual matter species. Note that

$$
h^{\prime}=\frac{1}{3} k \mathcal{Z}-\mathcal{H} A
$$

and that the $\kappa$ appearing above is the bare (not necessarily the measured) one. Furthermore, for convenience we define the frame-independent (FI) variables [18]

$$
\begin{aligned}
\tilde{q} & =q+(\rho+p) \sigma, \\
\tilde{x} & =æ+\sigma,
\end{aligned}
$$

$\tilde{æ}$ is FI according to Eq. (33) because we know that the anisotropic pressure tensor $\pi_{a b}$ is frame invariant. Hence, it follows from Eq. (13), that $\tilde{q}$ is also FI up to first order in perturbation. In the zero-shear frame (the Newtonian gauge), we have simply $\tilde{q}=q$ and $\tilde{e}=æ$.

Before presenting the evolution equations, we first write the dynamical quantities of the $Æ$-field in terms of the harmonic coefficients introduced above:

$$
\begin{aligned}
& \kappa \mathcal{X}^{\circledR} a^{2}=\alpha k \mathcal{H}(\mathcal{Z}+æ) \\
& -c_{14}\left[k^{2} A+k\left(æ^{\prime}+\mathcal{H} æ\right)\right], \\
& \kappa \mathcal{X}^{p, \circledast} a^{2}=-\frac{\alpha}{3} k\left[(\mathcal{Z}+æ)^{\prime}+2 \mathcal{H}(\mathcal{Z}+æ)\right] \\
& -\alpha\left(\mathcal{H}^{\prime}-\mathcal{H}^{2}\right) A \\
& \kappa q^{\circledR} a^{2}=-\frac{1}{3} \alpha k^{2}(\mathcal{Z}+æ)-\frac{2}{3} c_{13} k^{2} \tilde{\propto}, \\
& \kappa \Pi^{\circledR} a^{2}=c_{13} k\left(\tilde{\dddot{x}}^{\prime}+2 \mathcal{H} \tilde{\dddot{x}}\right) \text {. }
\end{aligned}
$$

In these expressions we have used both æ and $\tilde{\gtrless}$ because not all these quantities are FI. However it can be shown 
that the two quantities

$$
\begin{aligned}
& \kappa a^{2}\left(\mathcal{X}^{p, \circledast}-\frac{p^{\prime}}{\rho^{\prime}} \mathcal{X}^{\circledast}\right) \\
& =\alpha\left[\frac{1}{2} \kappa a^{2}\left(\mathcal{X}^{p}-\frac{p^{\prime}}{\rho^{\prime}} \mathcal{X}\right)-k^{2} \frac{p^{\prime}}{\rho^{\prime}} \Phi+\frac{\Xi^{\prime}}{3 \mathcal{H} \Xi} \kappa a^{2} \Pi\right] \\
& -\left(\frac{1}{3}+\frac{\Xi^{\prime}}{3 \mathcal{H} \Xi}\right) c_{14}\left(k \tilde{x}^{\prime}+k \mathcal{H} \tilde{\dddot{x}}-k^{2} \Phi\right) \\
& -\frac{1}{3} \alpha k \tilde{\dddot{x}}^{\prime}-\frac{1}{3} \alpha k \mathcal{H} \tilde{x}+\frac{\Xi^{\prime}}{3 \mathcal{H} \Xi} \alpha k \mathcal{H} \tilde{\dddot{x}}
\end{aligned}
$$

and

$$
\begin{aligned}
\kappa a^{2} \tilde{q}^{\mathbb{E}} & \equiv \kappa a^{2}\left[\left(\rho^{\mathbb{E}}+p^{\mathbb{E}}\right) \sigma+q^{\mathbb{E}}\right] \\
& =\frac{1}{2} \alpha \kappa a^{2} \tilde{q}-\frac{1}{3} \alpha k^{2} \tilde{\nsupseteq}-\frac{2}{3} c_{13} k^{2} \tilde{x},
\end{aligned}
$$

which will be used in the derivations, are FI, as they are expressed in terms of FI variables only. Note that in the above we have defined $\Phi \equiv \phi-\frac{\kappa a^{2}}{2 k^{2}} \Pi$ for convenience, where $\Phi$ is the Newtonian gravitational potential, and

$$
\Xi \equiv \frac{1}{2} \kappa a^{2}(\rho+p)=\mathcal{H}^{2}-\mathcal{H}^{\prime}, \quad p^{\prime} / \rho^{\prime}=-\frac{1}{3}-\frac{\Xi^{\prime}}{3 \mathcal{H} \Xi} .
$$

We now investigate in detail Eqs. (55, 56). On large scales, where $k \tau \ll 1$, the terms involving $k$ (these include the $\Pi$ term in Eq. (55D) can be safely disregarded, and as a result we have

$$
\begin{aligned}
\kappa\left(\mathcal{X}^{p, \circledast}-\frac{p^{\prime Æ}}{\rho^{\prime Æ}} \mathcal{X}^{\boxplus}\right) & \simeq \frac{\frac{1}{2} \alpha}{1-\frac{1}{2} \alpha} \kappa\left(\mathcal{X}^{p, f}-\frac{p^{\prime f}}{\rho^{\prime f}} \mathcal{X}^{f}\right)(57) \\
\kappa \tilde{q}^{E} & \simeq \frac{\frac{1}{2} \alpha}{1-\frac{1}{2} \alpha} \kappa \tilde{q}^{f}
\end{aligned}
$$

where the superscript ${ }^{f}$ means the fluid matter. We see that on large scales these attributes of the $\mathbb{E}$-field track those for other matter species in the universe. As the combination $\mathcal{X}^{p, f}-\frac{p^{\prime}}{\rho^{\prime}} \mathcal{X}^{f}$ determines the type of perturbations (for example, the perturbation is adiabatic if the combination is equal to zero), this indicates that the $\mathbb{E}$ field will not alter the type of the scalar perturbation produced in the inflationary era. Note that the above tracking behaviors are the same as that in the background cosmology, i.e., rescaling the gravitational constant by a same factor.

Now we can proceed to derive the evolution equations for our $Æ$-model. The propagation equation for the $Æ$ -field Eq. (28), in terms of the FI variables, becomes

$$
\begin{aligned}
\left(c_{14}-c_{13} \alpha\right) \epsilon^{\prime \prime}-\alpha\left(1+c_{13}\right)\left(\mathcal{H}^{\prime}-\mathcal{H}^{2}\right) \epsilon & (59) \\
+\frac{\alpha+2 c_{13}}{3} k^{2} \epsilon-\left(\alpha+c_{14}\right) k(a \Phi)^{\prime}-\frac{\alpha}{k} a\left(\kappa \Pi^{f} a^{2}\right)^{\prime} & =0
\end{aligned}
$$

in which we have changed the variable to $\epsilon \equiv a \tilde{x}$ for simplicity. Taking the time derivative of Eq. (43), adding to it $\left(4+3 \frac{p^{\prime}}{\rho^{\prime}}\right) \mathcal{H}$ times the same equation, and using Eq. (46) to eliminate the $q^{\prime}$ term, we obtain the following equation second-order differential equation for $\Phi$ :

$$
\begin{aligned}
& \Phi^{\prime \prime}+\left(2 \mathcal{H}-\frac{\Xi^{\prime}}{\Xi}\right) \Phi^{\prime}+\left(2 \mathcal{H}^{\prime}-\frac{\Xi^{\prime}}{\Xi} \mathcal{H}\right) \Phi+k^{2} \frac{p^{\prime}}{\rho^{\prime}} \Phi \\
= & \frac{1}{2} \kappa a^{2}\left(\mathcal{X}^{p}-\frac{p^{\prime}}{\rho^{\prime}} \mathcal{X}\right)-\frac{1}{k^{2}} \kappa a^{2}\left[\Pi^{\prime \prime}+\left(5-\frac{\Xi^{\prime}}{\mathcal{H} \Xi}\right) \mathcal{H} \Pi^{\prime}+2 \mathcal{H}^{\prime} \Pi+\left(6-2 \frac{\Xi^{\prime}}{\mathcal{H} \Xi}\right) \mathcal{H}^{2} \Pi-\frac{\Xi^{\prime}}{3 \mathcal{H} \Xi} k^{2} \Pi\right] .
\end{aligned}
$$

Eqs. (59, 60) are the evolution equations for the coupled $\epsilon-\Phi$ system that we are looking for (note that $\Pi=$ $\Pi^{Æ}+\Pi^{f}$ where $\Pi^{f}$ is the fluid matter anisotropic stress and $\Pi^{Æ}$ can also be expressed in terms of $\epsilon$ and its time derivatives by virtue of Eq. (54)). They are not closed if $\mathcal{X}^{p, f}-\frac{p^{\prime f}}{\rho^{\prime f}} \mathcal{X}^{f}$ and $\Pi^{f}$ are unknown and to know these quantitie we would need to know the matter content of the universe.

\section{The Primordial Power Spectra}

In the analysis above, we have mentioned that the pres-

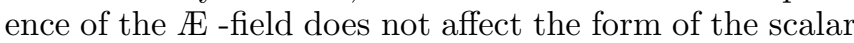
perturbation produced during inflation. But we also need to know whether other features of the inflationary power spectrum, such as the spectral index and the amplitude, are modified by the $Æ$-field, as compared with the predictions in standard GR. Here, we will investigated this issue (see [16] for a study in the absence of the $c_{4}$ term in the $\rightleftarrows$-Lagrangian) by considering a single-field model

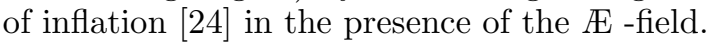


During the inflationary epoch a scalar inflaton field $\varphi$ slowly rolls along its potential and has an almost constant energy density which drives an almost exponential expansion of the universal scale factor. The comoving Hubble length (the horizon) decreases with time so that in this process the quantum vacuum fluctuations of the inflaton field $\varphi$ on the scales of interest to us leave the horizon (their scales become larger than the horizon). The curvature perturbations they generate remain constant during their subsequent super-horizon evolution, until these scales eventually reenter the horizon long after the inflationary period has ended. During the radiation-dominated era when these modes stay outside the horizon, the metric perturbation $\Phi$ becomes a constant, which drives the density perturbations of different matter species, and leads to the observed CMB and matter power spectra after horizon re-entry.

There are no couplings between the scalar field and

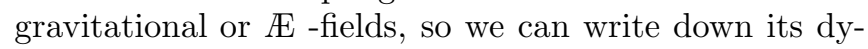
namical quantities for the scalar field $\varphi$ as

$$
\begin{aligned}
\rho^{\varphi} & =\frac{1}{2} \dot{\varphi}^{2}+V(\varphi), \\
p^{\varphi} & =\frac{1}{2} \dot{\varphi}^{2}-V(\varphi), \\
q_{a}^{\varphi} & =\dot{\varphi} \hat{\nabla}_{a} \varphi \\
\Pi_{a b}^{\varphi} & =0 .
\end{aligned}
$$

Making the following harmonic expansion for $\tilde{\nabla}_{a} \varphi$

$$
\hat{\nabla}_{a} \varphi=\sum_{k} \frac{k}{a} \chi Q_{a}^{k}
$$

it is easy to get

$$
\begin{aligned}
\mathcal{X}^{\varphi} & =\frac{1}{a^{2}}\left(\varphi^{\prime} \chi^{\prime}+\varphi^{\prime 2} A+a^{2} V_{\varphi} \chi\right), \\
\mathcal{X}^{p, \varphi} & =\frac{1}{a^{2}}\left(\varphi^{\prime} \chi^{\prime}+\varphi^{\prime 2} A-a^{2} V_{\varphi} \chi\right), \\
q^{\varphi} & =\frac{1}{a^{2}} k \varphi^{\prime} \chi
\end{aligned}
$$

where $V_{\varphi} \equiv \partial V(\varphi) / \partial \varphi$. Then parallel to Eqs. (55, 56) we have, following the standard procedure,

$$
\begin{aligned}
& a^{2}\left(\mathcal{X}^{p, \varphi}-\frac{p^{\prime}}{\rho^{\prime}} \mathcal{X}^{\varphi}\right) \\
= & \frac{4}{3}\left(1+\frac{\Xi^{\prime}}{4 \mathcal{H} \Xi}\right)\left[\varphi^{\prime} \tilde{\chi}^{\prime}-\varphi^{\prime 2} \Phi+a^{2} V_{\varphi} \tilde{\chi}+3 \mathcal{H} \varphi^{\prime} \tilde{\chi}\right](66)
\end{aligned}
$$

and

$$
\begin{aligned}
\kappa a^{2} \tilde{q}^{\varphi} & =\kappa\left[q^{\varphi}+\left(\rho^{\varphi}+p^{\varphi}\right) \sigma\right] \\
& =\kappa k \varphi^{\prime} \tilde{\chi}
\end{aligned}
$$

where we have defined the FI variable

$$
\tilde{\chi} \equiv \chi+\frac{\varphi^{\prime}}{k} \sigma .
$$

Substituting Eqs. (55, 67) into Eq. (60), and using Eq. (41) to eliminate the term proportional to $\varphi^{\prime} \tilde{\chi}^{\prime}-$ $\varphi^{\prime} \Phi+a^{2} V_{\varphi} \tilde{\chi}+3 \mathcal{H} \varphi^{\prime} \tilde{\chi}$, we arrive at the following equation

$$
\begin{aligned}
& \Phi^{\prime \prime}+\left(2 \mathcal{H}-\frac{\Xi^{\prime}}{\Xi}\right) \Phi^{\prime}+\left(2 \mathcal{H}^{\prime}-\frac{\Xi^{\prime}}{\Xi} \mathcal{H}\right) \Phi+\frac{1}{1-\frac{1}{2} \alpha}\left(1+\frac{1}{2} c_{14}\right) k^{2} \Phi \\
= & \frac{1}{1-\frac{1}{2} \alpha}\left[\frac{1}{2} c_{14}-\frac{1}{2}\left(c_{1}+c_{2}+c_{3}\right)-c_{13}\right] \frac{k}{a} \epsilon^{\prime}+\frac{1}{1-\frac{1}{2} \alpha}\left(c_{1}+c_{2}+c_{3}\right) \frac{\Xi^{\prime}}{2 \Xi} \frac{k}{a} \epsilon-\frac{1}{k} c_{13}\left(\frac{\epsilon^{\prime \prime \prime}}{a}-\frac{\Xi^{\prime}}{\Xi} \frac{\epsilon^{\prime \prime}}{a}-\Xi \frac{\epsilon^{\prime}}{a}\right) .
\end{aligned}
$$

Eqs. (59, 69) that form a closed set of evolution equations for the coupled $\circledast$-inflaton system; they are a generalization of the results presented in [16], and from them we can perform our analysis of the observational effects.

Let us look first at the large-scale evolution of $\Phi$. In this limit $k \tau \ll 1$ so the last term on the left-hand side, and the first two terms on the right hand side, of Eq. (69) can be dropped. Notice that during the inflationary era $\mathcal{H}^{2}-\mathcal{H}^{\prime}=\frac{1}{2} \kappa a^{2}(\rho+p) \simeq 0$ and Eq. (59) become

$$
\left(c_{14}-c_{13} \alpha\right) \epsilon^{\prime \prime}=\left(\alpha+c_{14}\right) k(a \Phi)^{\prime} .
$$

Substituting this into Eq. (69) one could see that

$$
\begin{aligned}
& \frac{1}{k} c_{13}\left(\frac{\epsilon^{\prime \prime \prime}}{a}-\frac{\Xi^{\prime}}{\Xi} \frac{\epsilon^{\prime \prime}}{a}-\Xi \frac{\epsilon^{\prime}}{a}\right) \\
\propto & \Phi^{\prime \prime}+\left(2 \mathcal{H}-\frac{\Xi^{\prime}}{\Xi}\right) \Phi^{\prime}+\left(2 \mathcal{H}^{\prime}-\frac{\Xi^{\prime}}{\Xi} \mathcal{H}\right) \Phi
\end{aligned}
$$

so that we finally have

$$
\Phi^{\prime \prime}+\left(2 \mathcal{H}-\frac{\Xi^{\prime}}{\Xi}\right) \Phi^{\prime}+\left(2 \mathcal{H}^{\prime}-\frac{\Xi^{\prime}}{\Xi} \mathcal{H}\right) \Phi=0
$$

on super-horizon scales. Note that one should not simply set $\epsilon^{\prime \prime}=0$ in this limit as was done in Ref. [16], although that leads to the same result [28]. This equation is still 
valid outside the inflationary epoch within the parameter space given by Eq. (38).

The solution to Eq. (71) is given by

$$
\Phi=D\left(1-\frac{\mathcal{H}}{a^{2}} \int a^{2} d \tau\right)
$$

where $D$ is a constant. Long after leaving the horizon, the potential $\Phi$ and the scalar perturbation $\tilde{\chi}$ have time to evolve, and after inflation $\tilde{\chi}$ ceases to exist. However, as mentioned above, during the radiation era on super-horizon scales $\Phi$ finally becomes a constant which is related to $D$ by

$$
\Phi=\frac{2}{3} D .
$$

Once $D$ is known we could set the initial conditions of $\Phi$ in the radiation era for the subsequent evolutions. The quantity $D$ could be fixed by matching to the inflaton perturbation $\tilde{\chi}$ at the time of horizon exit $(k=a H=\mathcal{H})$ as follows: substituting Eqs. (56, 67) into Eq. (43) we get

$$
\begin{aligned}
k(a \Phi)^{\prime}= & \frac{1}{1-\frac{1}{2} \alpha}\left[\frac{1}{2} k a \kappa \varphi^{\prime} \tilde{\chi}-\frac{1}{2}\left(c_{1}+c_{2}+c_{3}\right) k^{2} \epsilon\right] \\
& -c_{13}\left[\epsilon^{\prime \prime}+\left(\mathcal{H}^{\prime}-\mathcal{H}^{2}\right) \epsilon\right] .
\end{aligned}
$$

Now we begin to confine ourselves within the parameter space Eq. (37). In that case $c_{14}=-\alpha=\left(c_{1}+c_{3}\right)\left(c_{1}-\right.$ $\left.c_{3}\right) / c_{1}$ and the $\mathbb{E}$-field EOM Eq. (59) becomes

$$
\alpha\left(1+c_{13}\right)\left[\epsilon^{\prime \prime}+\left(\mathcal{H}^{\prime}-\mathcal{H}^{2}\right) \epsilon\right]=\left(c_{1}+c_{2}+c_{3}\right) k^{2} \epsilon .
$$

So, from these two equations we find that

$$
(a \Phi)^{\prime}=\frac{1}{1-\frac{1}{2} \alpha} \frac{1}{2} a \kappa \varphi^{\prime} \tilde{\chi} .
$$

On the other hand, from Eq. (72) one can write

$$
(a \Phi)^{\prime}=\frac{1}{2} \frac{1}{1-\frac{1}{2} \alpha} \kappa a \varphi^{\prime 2} \frac{1}{\mathcal{H}} D .
$$

Obviously, matching these two expressions gives the value of $D$

$$
D=\frac{\mathcal{H}}{\varphi^{\prime}} \tilde{\chi}
$$

where the three variables $\mathcal{H}, \varphi^{\prime}$ and $\tilde{\chi}$ are all evaluated at the horizon exit. As a result, the initial power spectrum for $\Phi, \mathcal{P}_{\Phi}=k^{3}\left\langle\Phi^{2}\right\rangle / 2 \pi^{2}$ where $\langle\cdots\rangle$ means the ensemble average, is given by

$$
\begin{aligned}
\mathcal{P}_{\Phi} & =\frac{4}{9} \frac{\mathcal{H}^{2}}{\varphi^{\prime 2}}\left\langle\tilde{\chi}^{2}\right\rangle \\
& =\frac{4}{9} \frac{\mathcal{H}^{2}}{\varphi^{\prime 2}}\left(\frac{H}{2 \pi}\right)^{2}
\end{aligned}
$$

in which we have used the relation $\mathcal{P}_{\tilde{\chi}}=k^{3}\left\langle\tilde{\chi}^{2}\right\rangle / 2 \pi^{2}=$ $(H / 2 \pi)^{2}$ [24] (as discussed in Ref. [16], this relation is not affected by the $Æ$-field up to first order because the Æ -field is not coupled to the inflaton) and the Hubble expansion rate $H=\dot{a} / a$ is also evaluated at the time of horizon exit.

Note that the $\Phi$ power spectrum Eq. (79) has exactly the same form as in standard GR. To compare their magnitudes, let us write

$$
\frac{\mathcal{P}_{\Phi}^{\mathrm{E}}}{\mathcal{P}_{\Phi}^{\mathrm{GR}}}=\frac{\left(H^{2} / \varphi^{\prime 2}\right)_{\circledast}}{\left(H^{2} / \varphi^{\prime 2}\right)_{\mathrm{GR}}}
$$

in which we have used the fact that at the time of horizon exit $\mathcal{H}=k$ should be the same in the two models. If we further assume the same inflation potential, then 20] because the background expansion in our Æther model is indistinguishable from that of GR, we have

$$
\frac{\mathcal{P}_{\Phi}^{\mathbb{E}}}{\mathcal{P}_{\Phi}^{\mathrm{GR}}}=1
$$

that is, the power spectra in these two models are exactly the same, in both the shape and the magnitude. It should be noticed that if the background evolution, $H$, of the Æ-model during the inflation is different from GR, then the evolutions of $\varphi$ will also be different because of the scalar field equation of motion; as a result the slow-roll parameters at the horizon crossing are generally different in these two models and so will be the spectral indices.

What is the fate of the vector and tensor modes of the Æ -field perturbation? As shown in [16], the vector mode is also sourceless unless exotic matter such as cosmological defects exist. Hence, it should decay similarly all the way up to the present and leave no traces in the $\mathrm{CMB}$ observables. As for the tensor modes, from Eqs. (29- 33)

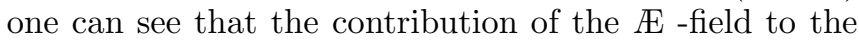
tensor modes lies only in $\kappa \Pi^{\mathbb{E}}$ and is independent of the model parameter $c_{4}$. Consequently, the power spectrum of the tensor perturbations given in [16]

$$
\mathcal{P}_{h}=\kappa\left(1+c_{13}\right)^{1 / 2} \frac{H^{2}}{2 \pi^{2}}
$$

is still valid here (note that what appears in this formula is the true bare gravitational constant). Following the argument above again, we can show that

$$
\frac{\mathcal{P}_{h}^{Æ}}{\mathcal{P}_{h}^{\mathrm{GR}}}=\left(1+\frac{1}{2} c_{14}\right)\left(1+c_{13}\right)^{1 / 2} .
$$

According to Eq. (38),,$c_{13}<0$ and $c_{14}<0$, so the amplitude of the gravitational-wave spectrum predicted in our $\rightleftarrows$-model is smaller than that arising in standard GR. Although this may provide a discrimination between the two models, the difficulty in observing the tensor spectrum will be a great hurdle for the use of this discriminator: if our Universe turns out to be described by the $\rightleftarrows$-model, then this spectrum is even more difficult to observe than in GR. 


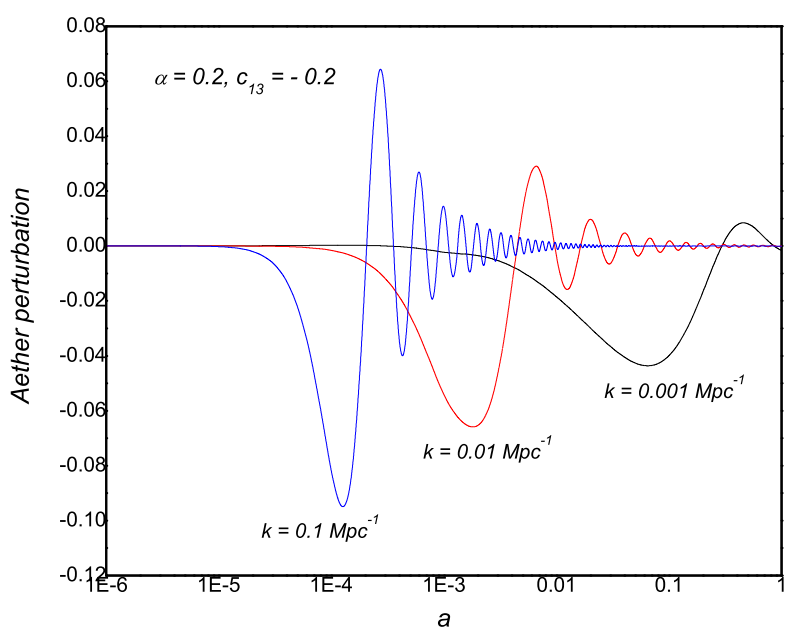

FIG. 1: (Color online) The evolution of the $Æ$-field perturbation $\tilde{æ}$ versus the cosmic scale factor $a$. We have shown it for 3 different scales $k=0.1,0.01,0.001 \mathrm{Mpc}^{-1}$ respectively, as indicated beside the curves. The model parameters are $\alpha=0.2, c_{13}=-0.2$, which satisfy the constraints Eq. (38).

\section{The $\rightleftarrows$-Effects on Late-time Cosmology}

We have seen in the above that in our $Æ$-model the primordial scalar-mode power spectrum is exactly the same as assuming standard GR and using the measured value $\kappa_{\star}$ in calculations. The next question is if possible deviations from GR will emerge in the subsequent evolutions of the perturbations. Interestingly, we find that the answer is yes. The reason is that, although the $\mathbb{E}$-perturbation is sourceless during the inflationary era, in subsequent stages where the fluid anisotropic stresses are nonzero it becomes sourced. To see the consequences explicitly, we now write the $\mathbb{E}$-field EOM within the parameter space Eq. (37) as

$$
\alpha\left[\left(1+c_{13}\right) k\left(\tilde{\mathfrak{x}}^{\prime}+2 \mathcal{H} \tilde{\dddot{x}}\right)+\kappa \Pi^{f} a^{2}\right]^{\prime}=\frac{\alpha+2 c_{13}}{3} k^{3} \tilde{œ} .
$$

During the inflationary epoch $\Pi^{f}=0$ and $\mathcal{H}^{\prime}-\mathcal{H}^{2} \simeq 0$, thus the solution to the above equation is

$$
\tilde{x}(\tau) \simeq \frac{A_{1}}{a} \sin \left(c_{s} k \tau\right)+\frac{A_{2}}{a} \cos \left(c_{s} k \tau\right),
$$

where we have defined $c_{s}^{2} \equiv \frac{c_{13}}{3\left(1+c_{13}\right)\left(c_{1}-c_{3}\right)}$ which is positive according to Eq. (38); $A_{1,2}$ are integration constants. Meanwhile, during this epoch the scale factor $a$ undergoes exponential growth, which means that $\tilde{x}$ decays exponentially and its initial value is washed out soon.

In the radiation dominated epoch, $a \propto \tau$ and $\Pi^{f}=$ $\Pi^{\gamma}+\Pi^{\nu} \neq 0$ (here subscripts $\gamma,{ }_{\nu}$ denotes photon and neutrino respectively). Now the homogeneous part of the Æ -field EOM becomes

$$
\tilde{\mathfrak{X}}^{\prime \prime}+\frac{2}{\tau} \tilde{\mathfrak{X}}^{\prime}-\frac{2}{\tau^{2}} \tilde{\dddot{X}}+c_{s}^{2} k^{2} \tilde{\dddot{X}}=0
$$

whose solution is

$$
\begin{aligned}
\tilde{æ}_{\text {gen }}(\tau)= & \frac{B_{1}}{\tau^{2}}\left[\cos \left(c_{s} k \tau\right) c_{s} k \tau-\sin \left(c_{s} k \tau\right)\right] \\
& +\frac{B_{2}}{\tau^{2}}\left[\sin \left(c_{s} k \tau\right) c_{s} k \tau+\cos \left(c_{s} k \tau\right)\right]
\end{aligned}
$$

where $B_{1,2}$ are integration constants and a subscript gen means the general solution. We can see that in the limit $c_{s} k \tau \lesssim 1 \tilde{æ}_{\text {gen }}$ decays as $\tilde{æ}_{\text {gen }} \sim 1 / \tau^{2}$ and when $c_{s} k \tau \gg 1$ it decreases as $\tilde{\nexists g}_{\text {gen }} \sim B_{1} \cos \left(c_{s} k \tau\right) / \tau+B_{2} \sin \left(c_{s} k \tau\right) / \tau$. Meanwhile there is a particular solution of $\approx$ which involves a weighted integration of $\left(\kappa \Pi^{f} a^{2}\right)^{\prime}$ over time. Deep into the radiation-dominated epoch, the decaying general solutions of $\tilde{\propto}$ have become negligible and the growing particular solution is still tiny, so we can reasonably take the initial conditions of $\tilde{æ}$ as $\tilde{æ}_{\text {ini }}=\tilde{æ}_{i n i}^{\prime}=0$ in our numerical calculations. We have checked that, for the scales we are interested in, this choice of initial conditions is robust and the numerical results are not sensitive to (not dramatically) different initial conditions, and in Fig. 1 1we have depicted the time evolution of $\tilde{x}$ at different scales (or different $k$ ) for the model $\alpha=-c_{13}=0.2$. It can be seen there that, at early times $\tilde{æ}$ remains close to zero; later it grows as $\left(\kappa \Pi^{f} a^{2}\right)^{\prime}$ deviates significantly from 0 , and finally when $\left(\kappa \Pi^{f} a^{2}\right)^{\prime}$ becomes tiny in the matterdominated era it undergoes a (oscillatory) decay again.

Now what kind of signatures will this behavior of $\tilde{æ}$ imprint on the perturbation evolution? In order to answer this question, we find it useful to rewrite the total density perturbation, pressure perturbation, heat flux and anisotropic stress (with the contributions from the Æfield included) as follows:

$$
\begin{aligned}
\kappa \mathcal{X} a^{2} & =\kappa \mathcal{X}_{\mathrm{tr}} a^{2}+\kappa \mathcal{X}_{\mathrm{ntr}} a^{2} \\
\kappa \mathcal{X}^{p} a^{2} & =\kappa \mathcal{X}_{\mathrm{tr}}^{p} a^{2}+\kappa \mathcal{X}_{\mathrm{ntr}}^{p} a^{2}, \\
\kappa q a^{2} & =\kappa q_{\mathrm{tr}} a^{2}+\kappa q_{\mathrm{ntr}} a^{2} \\
\kappa \Pi a^{2} & =\kappa \Pi_{\mathrm{tr}} a^{2}+\kappa \Pi_{\mathrm{ntr}} a^{2}
\end{aligned}
$$

where the quantities with subscript tr

$$
\begin{aligned}
\kappa \mathcal{X}_{\mathrm{tr}} a^{2} & =\kappa_{\star} \mathcal{X}^{f} a^{2}, \\
\kappa \mathcal{X}_{\mathrm{tr}}^{p} a^{2} & =\kappa_{\star} \mathcal{X}^{p, f} a^{2}, \\
\kappa q_{\mathrm{tr}} a^{2} & =\kappa_{\star} q^{f} a^{2}, \\
\kappa \Pi_{\mathrm{tr}} a^{2} & =\kappa_{\star} \Pi^{f} a^{2}
\end{aligned}
$$

are the tracking parts because they are exact rescalings of the corresponding quantities for the fluid matter [we remind that $\kappa_{\star}=\kappa /\left(1-\frac{1}{2} \alpha\right)$ ], while those with subscript 
ntr are the non-tracking parts expressed as

$$
\begin{aligned}
& \kappa \mathcal{X}_{\mathrm{ntr}} a^{2}=\alpha \kappa_{\star} \Pi^{f} a^{2} \\
& +\frac{\alpha}{1-\frac{1}{2} \alpha}\left(1+c_{13}\right) k\left(\tilde{æ}^{\prime}+2 \mathcal{H} \tilde{\dddot{x}}\right), \\
& \kappa \mathcal{X}_{\mathrm{ntr}}^{p} a^{2}=-\frac{1}{3} \alpha \kappa_{\star} \Pi^{f} a^{2} \\
& -\frac{1}{3} \frac{\alpha}{1-\frac{1}{2} \alpha}\left(1+c_{13}\right) k\left(\tilde{\mathfrak{x}}^{\prime}+2 \mathcal{H} \tilde{\mathfrak{x}}\right), \\
& \kappa q_{\mathrm{ntr}} a^{2}=-\frac{1}{3} \frac{\alpha+2 c_{13}}{1-\frac{1}{2} \alpha} k^{2} \tilde{æ}, \\
& \kappa \Pi_{\mathrm{ntr}} a^{2}=c_{13} k\left(\tilde{\mathfrak{x}}^{\prime}+2 \mathcal{H} \tilde{\dddot{x}}\right)-\frac{1}{2} \alpha \kappa_{\star} \Pi^{f} a^{2} ;
\end{aligned}
$$

only the measured value $\kappa_{\star}$ appears in the final expressions. It can be easily checked that the tracking and non-tracking parts satisfy the energy momentum conservation equation separately by utilizing the $\rightleftarrows$-field EOM. In particular, the non-tracking part effectively represents a purely perturbed contribution to the total energy momentum tensor that has no background counterparts. We also note that $\kappa \mathcal{X}_{\mathrm{ntr}}^{p} a^{2}=-\frac{1}{3} \kappa \mathcal{X}_{\mathrm{ntr}} a^{2}$.

Taking the time derivative of Eq. (44), using Eq. (45) to eliminate the $\eta^{\prime}$ term and cancelling the $\mathcal{X}^{\prime}$ appearing there with Eq. (47), one arrives at the following evolution equation of $\mathcal{Z}$ :

$$
k\left(\mathcal{Z}^{\prime}+\mathcal{H} \mathcal{Z}\right)+\frac{1}{2} \kappa\left(\mathcal{X}+3 \mathcal{X}^{p}\right) a^{2}=0
$$

or equally

$$
k\left(\mathcal{Z}^{\prime}+\mathcal{H} \mathcal{Z}\right)+\frac{1}{2} \kappa_{\star}\left(\mathcal{X}^{f}+3 \mathcal{X}^{p, f}\right) a^{2}=0
$$

that is, the non-tracking part does not contribute to the evolution of $\mathcal{Z}$. In this subsection we work in the CDM frame where the 4-acceleration $A=0$, and thus $\Delta_{\mathrm{CDM}}^{\prime}=-k \mathcal{Z}$ [18] (where $\Delta \equiv \mathcal{X} / \rho$ is the density contrast) which means the growth of matter perturbation is not affected by the non-tracking part. However, since $\kappa \mathcal{X}_{\text {ntr }} a^{2}$, which is nonzero at early times, appears in Eq. (44), the values of $\kappa_{\star} \mathcal{X}^{f} a^{2}$ and $\mathcal{Z}$ in this equation are slightly modified that change the subsequent evolution of $\mathcal{Z}$ a little. These can be seen in Fig. 2, where we have displayed the matter power spectrum of the $Æ$-model for different choices of parameters. Obviously the spectrum depends weakly on the parameters; furthermore, its shape is essentially the same as that in $\Lambda$ CDM model so that the parameters will degenerate with the bias relating the matter power spectrum to the observed spectrum of galaxies. In addition to that, there is also the degeneracy with respect to the neutrino masses, since the latter affects its anisotropic stress and the time at which they become non-relativistic. Taken together this indicates that the data for $P(k)$ will not place very stringent con-

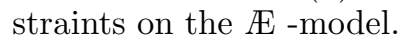

From Eqs. 41- 43) one can see that $\phi, \sigma^{\prime}, \phi^{\prime}$ are also influenced by the non-tracking part, and because these

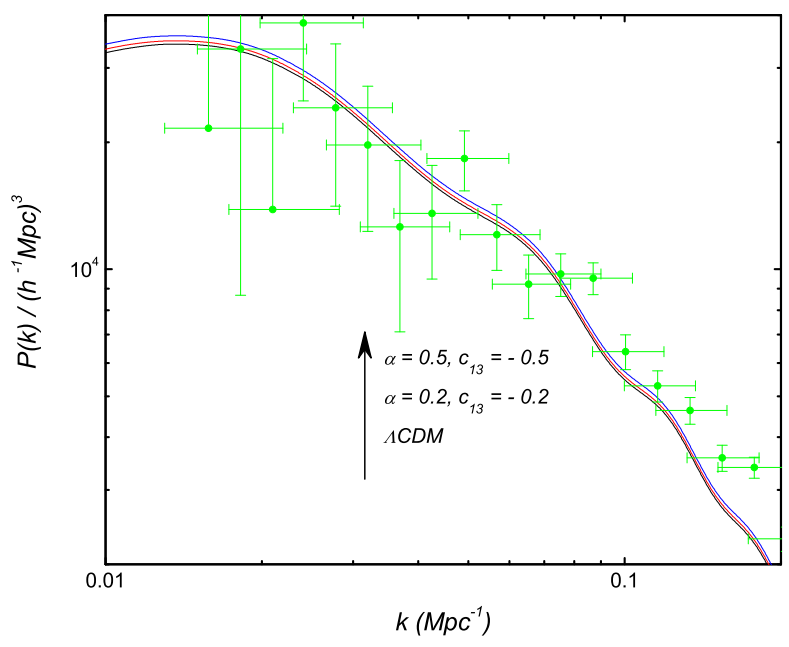

FIG. 2: (Color online) The matter power spectrum of the $\rightleftarrows$-model considered in this work. From top to bottom the curves correspond to $\left(\alpha, c_{13}\right)=(0.5,-0.5),(0.2,-0.2)$ and $(0,0)$ (which is just $\Lambda \mathrm{CDM}$ ) respectively. The other parameters common for all curves are $\Omega_{b} h^{2}=0.0223, \Omega_{c} h^{2}=0.1054$ and $h=0.732$ where $\Omega_{b, c}$ are the fractional energy density of baryons and CDM, and $h \equiv H_{0} /(100 \mathrm{~km} / \mathrm{s} / \mathrm{Mpc})$. The differences among these spectra are small although their parameters are quite different.

variable determine the CMB power spectrum, we could expect the latter to be modified by the presence of the $\rightleftarrows$ -field as well. This is correct, as shown Fig. 3. where we have plotted the CMB spectra for different choices of the model. Obviously the CMB power spectrum is changed by the $\rightleftarrows$-field, though this effect is not very strong and so it might be difficult to place stringent limits on the parameter space of the theory.

There are two points that need to be noticed. Firstly, in this work we are assuming that the recent cosmic acceleration is caused by a cosmological constant, the anisotropic stress of which is zero. If in contrast the dark energy has a nonzero anisotropic stress (as in [25, 26, 27]) then the $Æ$-field perturbation $\tilde{e}$ probably will not decay at late times, and this might lead to further modifications of the CMB power spectrum. Secondly, when $f(K)$ is not simply a linear function of $K$, the $\rightleftarrows$-field will be sourced by the evolution of the gravitational potential as well, and its effects will be more intricate and interesting. Such new features may appear in more complicated models like TeVeS [2, 5] and $Æ$-fields with non-canonical kinetic terms [6].

In total, these results indicate that within the parameter space Eq. (37) the background cosmology of our $\mathbb{E}$ -model is the same as in GR, while the CMB and matter power spectra differ slightly from the predications of GR. In principle, these features could be used to constrain the parameters $\alpha$ and $c_{13}$ of the present model. This will 


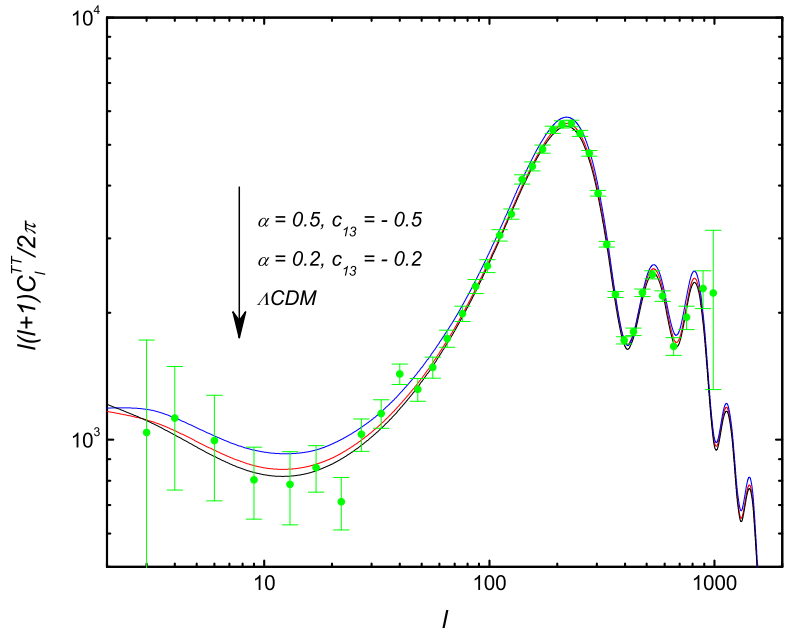

FIG. 3: (Color online) The CMB power spectrum of the $\rightleftarrows$ -model considered in this work. At $l \sim 10$ from top to bottom the curves correspond to $\left(\alpha, c_{13}\right)=(0.5,-0.5),(0.2,-0.2)$ and $(0,0)$ (which is just $\Lambda$ CDM) respectively. The other parameters common for all curves are $\Omega_{b} h^{2}=0.0223, \Omega_{c} h^{2}=0.1054$ and $h=0.732$ where $\Omega_{b, c}$ are the fractional energy density of baryons and CDM, and $h \equiv H_{0} /(100 \mathrm{~km} / \mathrm{s} / \mathrm{Mpc})$.

generally involve a full search of the parameter space using for example the Markov Chain Monte Carlo method, which is beyond the scope of this work. However, as we discussed above, the constraints from the linear spectra may not be very stringent anyway.

\section{DISCUSSION AND CONCLUSION}

In this paper we have studied the cosmology of the Einstein- $Æ$ ther theory. After presenting the general field equations for such theories in the CGI formalism, we focussed on a specific class of models described in Eq. (34), and confined ourselves to the parameter space of models described by Eq. (38) which pass the PPN and Cerenkov constraints. This parameter space is known to have the same locally- and cosmologically-felt gravitational constants $\left(\kappa_{0}=\kappa_{\infty}\right.$, which are different from the true bare $\kappa)$ and this tracking behavior indicates that we can consider its background expansion just by using the measured value $\kappa_{\star}=\kappa_{0}$ and ignoring the presence of the $\rightleftarrows$ -field (since the only effect of the Æther field is to track other matter species, the model in Eq. (34) clearly cannot explain dark energy. More general models, such as the $f(K)$ proposed in [6] and our Sec. II may serve this purpose).

We find that it is a general feature that the tracking behavior not only occurs at the background level but also at the linear order in perturbation theory. For example, the quantity $\mathcal{X}^{p, \circledast}-\frac{p^{\prime \star}}{\rho^{\prime 灬}} \mathcal{X}^{Æ}$ tracks that of the other matter species on super-horizon scales. This indicates that, whatever type of perturbation is generated during inflation, the presence of $Æ$-field will not alter it. In particular, in the single-field inflation model we consider, no isocurvature perturbation is produced. This is an important characteristic, and it would be interesting to see whether similar behavior occurs for general, higher-order choices of $f(K)$.

We generalized the analysis of primordial power spectra for the $\mathbb{E}$-theory [16] to our model. For the parameter ranges which satisfy local gravity bounds, we find that the evolution of the large-scale gravitational potential, $\Phi$, is unmodified as compared with that in GR, and show that the primordial power spectrum of $\Phi$ also has the same form as in GR [c.f. Eq. (79)]. If we assume that the bare gravitational constants in GR and in the $\mathbb{E}$ model are the same, then the magnitudes of the spectra are different in these two models. However, contrary to the discussion in [16], we argue that we do not know the true bare $\kappa$, but only know the measured value, $\kappa_{\star}=\kappa_{0}$. In both GR and our $\rightleftarrows$-model, $\kappa_{0}$ is equal to the cosmological value $\kappa_{\infty}$, while in the latter $\kappa \neq \kappa_{\infty}$. As a result, we show that with the same inflationary potential the primordial power spectra of $\Phi$ in these two models should have the same shape and the same magnitude. Meanwhile, we also find that the power spectrum of tensor perturbations in our model is smaller in magnitude than is predicted in GR, and so currently we cannot use it to distinguish between GR and the $Æ$-model.

For the late-time evolution of the perturbations, it is shown that, the $\circledast$-field perturbation is driven by the evolution of the anisotropic photon and neutrino stresses - the $\left[\kappa_{\star}\left(\Pi^{\gamma}+\Pi^{\nu}\right) a^{2}\right]^{\prime}$ term in its propagation equation - although it is sourceless during inflation. Therefore, it decays away exponentially during the inflationary era, grows again in the radiation-dominated epoch when $\left[\kappa_{\star}\left(\Pi^{\gamma}+\Pi^{\nu}\right) a^{2}\right]^{\prime}$ is significant, and finally diminishes again, oscillating when $\left[\kappa_{\star}\left(\Pi^{\gamma}+\Pi^{\nu}\right) a^{2}\right]^{\prime}$ eventually becomes negligible. As a result, the $\mathrm{CMB}$ and matter power spectra are modified by the existence of the $Æ$ -field. We also remark that, depending on the nature of the dark energy, it is possible that the $Æ$-field perturbation will have non-trivial dynamics driven by the $\left(\kappa_{\star} \Pi^{\mathrm{DE}} a^{2}\right)^{\prime}$ term, where $\Pi^{\mathrm{DE}}$ is the possible dark-energy anisotropic stress [25].

We also note that there recently appeared a later paper [29] which investigated in details the structure formation of the model proposed in [6]. In their Sec. V the authors considered a simple power-law model $f(K)=\gamma(-K)^{n}$ in which $n=1$ corresponds to our Lagrangian Eq. (34) (except for the $c_{4}$ term). They obtained criteria for there to be a growing mode in the evolution of the (scalar-mode) Æ-field perturbation. However their criteria cannot be applied directly to the model we consider here. To see why, note that in Eq. (32) of 29] the quantities $\Psi, \Phi$ also contain $V$ (their $V$ is equivalently our $\tilde{x}$ ). However, by their argument the $V$ terms in $\Psi, \Phi$ are suppressed by a small quantity $F_{K}$ (which in our notation is just $F$ ) 
and are ultimately neglected from the Æ-field equation of motion, their Eq. (43). In our model, there is no argument that $F \ll 1$ (its magnitude actually is 1 ) and so we can no longer neglect the $\tilde{æ}$ terms appearing in $\Phi, \Psi$. When these are taken into account we obtain a different equation of motion, i.e., the $b_{i}$ s in Eq. (43) of [29] are different in our work, and consequently the criteria for the existence of growing modes are different as well. In addition, are the facts that we have a $c_{4}$ term in our Lagrangian and use a different parameter space, Eqs. 37. [38), which also contribute to the differences between our results and those in [29]. As an aside, we stress that the key relation in our work, $c_{14}=-\alpha$, is a consequence of Eq. (37) and has nothing to do with whether or not the Æ-gravity waves propagate superluminally. In fact, we could do our calculation dropping the constraint that these waves propagate superluminally, and in this case the perturbation dynamics might place some constraints on the parameter space; for example, in some portion of the parameter space the growth of $\tilde{x}$ may become unstable. In our work we choose the parameters leading to superluminal gravity waves simply to avoid constraints from Cerenkov radiation. There are still some debates about this kind of choice, see however [10] for a different and conservative point of view.

There are also other general differences between the EA model we consider here and the GEA model of [29]. First, for the static and weak field limit, the $c_{4}$ term and the choice of parameter space Eq. (37) are crucial for the EA model to evade PPN tests [10]; in [29] there is no $c_{4}$ term, but the nonlinearity in the Æther field Lagrangian guarantees that the local gravitational tests are not a problem because at high densities the modification is negligible. Second, as we have seen above, our conclusion that the primordial power spectrum of density perturbation is unmodified compared with GR relies on the fact $c_{14}=-\alpha$ so that the background expansion during inflation in our model is the same as in GR (again the $c_{4}$ term is crucial here). If the $c_{4}$ term is not included, the local and cosmological gravitational constants are different, meaning that the background evolution during in- flation is different from GR; as a result the background quantities at horizon crossing, which determine the observables in inflationary models through the slow-roll parameters, may be different from those in GR - this means that the EA model will predict different shape of primordial density spectrum other than GR [30]. For the GEA model considered in [29], again the nonlinear $f(K)$ makes the modifications to GR during early times small enough to be neglected, and as such the primordial power spectrum is also the same as in GR. Note however that in the latter case [29] the late time evolution of vector field perturbation is also modified in a complex way, making the corresponding cosmological behaviors different from ours.

So, in conclusion, although the background expansion of our $\rightleftarrows$-model is exactly the same as that in GR, the cosmological data on $\mathrm{CMB}$ and matter power spectra can be used to constrain the $\rightleftarrows$-model. However, the constraint is not expected to be very stringent because the linear perturbation spectra depend weakly on the model parameters. Other considerations of the behaviour in strong gravitational fields, such as those studies of the compact stars or black holes [13, 14 would enable better constraints on the present model to be obtained. Alao, as general interests for cosmology, studies in more complicated Æ-field Lagrangian as those performed in [29, 31] need to be explored thoroughly.

\section{Acknowledgments}

We thank Ted Jacobson, Eugene Lim, Constantinos Skordis, Xiaoting Wang, HongSheng Zhao, Tom Zlosnik and the referee for helpful discussions. The numerical calculation in this work uses a modified version of the public CAMB code [32]. BL is supported by the Overseas Research Studentship, Cambridge Overseas Trust and the DAMTP at University of Cambridge. DFM acknowledges the Humboldt Foundation.
[1] M. Milgrom, Astrophys. J. 270, 365 (1983); ibid., Astrophys. J. 270, 371 (1983); ibid., Astrophys. J. 270, 384 (1983).

[2] J. D. Bekenstein, Phys. Rev. D70, 083509 (2004).

[3] C. Skordis et at., Phys. Rev. Lett. 96, 011301 (2006); C. Skordis, Phys. Rev. D74, 103513 (2006).

[4] S. Dodelson and M. Liguori, Phys. Rev. Lett. 97, 231301 (2006).

[5] T. G. Zlosnik, P. G. Ferreira and G. D. Starkman, Phys. Rev. D 74, 044037 (2006).

[6] T. G. Zlosnik, P. G. Ferreira and G. D. Starkman, Phys. Rev. D 75, 044017 (2007).

[7] C. Eling, T. Jacobson and D. Mattingly, arXiv: gr-qc/0410001

[8] Arianto, F. P. Zen, Bobby, E. Gunara, Triyanta and
Supardi (2007), arXiv:0709.3688 [hep-th]; S. Kanno and J. Soda, Phys. Rev. D 74, 063505 (2006); A. Tartaglia and N. Radicella (2007), arXiv:0708.0675 [gr-qc]; M. Libanov, V. Rubakov, E. Papantonopoulos, M. Sami and S. Tsujikawa, JCAP 0708, 010 (2007); P. G. Ferreira, B. M. Gripaios, R. Saffari and T. G. Zlosnik, Phys. Rev. D 75, 044014 (2007).

[9] T. Jacobson and D. Mattingly, Phys. Rev. D 64, 024028 (2001).

[10] B. Z. Foster and T. Jacobson, Phys. Rev. D 73, 064015 (2006).

[11] C. Bonvin, R. Durrer, P. G. Ferreira, G. Starkman and T. G. Zlosnik (2007), arXiv:0707.3519 [astro-ph].

[12] J. W. Elliott, G. D. Moore and H. Stoica, JHEP 08, 066 (2005). 
[13] C. Eling and T. Jacobson, Class. Quant. Grav. 23, 5625 (2006); C. Eling, T. Jacobson and M. C. Miller, Phys. Rev. D 76, 042003 (2007).

[14] C. Eling and T. Jacobson, Class. Quant. Grav. 23, 5643 (2006); R. A. Konoplya and A. Zhidenko, Phys. Lett. B 644, 186 (2007); D. Garfinkle, C. Eling and T. Jacobson, Phys. Rev. D 76, 024003 (2007); T. Tamaki and U. Miyamoto, arXiv: 0709.1011 [gr-qc].

[15] S. M. Carroll and E. A. Lim, Phys. Rev. D 70, 123525 (2004).

[16] E. A. Lim, Phys. Rev. D 71, 063504 (2005).

[17] A. Anaelle and H. Zhao (2007), arXiv: 0711.0958 [astro$\mathrm{ph}$.

[18] G. R. Ellis and M. Bruni, Phys. Rev. D 40, 1804 (1989); G. F. R. Ellis and H. Van Elst, in Theoretial and Observational Cosmology, ed. M. Lachièze-Rey (Springer, New York, 1998); A. Challinor and A. Lasenby, Astrophys. J. 513, 1 (1999); A. M. Lewis, PhD dissertation, Queens' College and Astrophysics Group, Cavendish Laboratory, Cambridge University, 2000.

[19] B. Li and M. C. Chu, Phys. Rev. D 74, 104010 (2006) [arXiv: astro-ph/0610486]; B. Li and J. D. Barrow, Phys. Rev. D 75, 084010 (2007) [arXiv: gr-qc/0701111; B. Li, K. C. Chan and M. C. Chu, Phys. Rev. D 76, 024002 (2007) [arXiv: astro-ph/0610794]; B. Li, J. D. Barrow and D. F. Mota, Phys. Rev. D 76, 044027 (2007) arXiv:0705.3795 [gr-qc]]; T. Koivisto and D. F. Mota, Phys. Lett. B 644, 104 (2007); T. Koivisto and D. F. Mota, Phys. Rev. D 75, 023518 (2007); B. Li, J. D. Barrow and D. F. Mota, Phys. Rev. D 76, 104047 (2007) (arXiv:0707.2664 [gr-qc]).

[20] We want to elaborate this point as it will also be used below. Suppose we have a universe described by either standard GR or the $\rightleftarrows$-theory Eq. (34) and we have a measured gravitational constant $\kappa_{\star}$. If we assume GR, then we have $\kappa_{0}^{\mathrm{GR}}=\kappa_{\star}=\kappa^{\mathrm{GR}}=\kappa_{\infty}^{\mathrm{GR}}$, while otherwise we have $\kappa_{0}^{\text {E }}=\kappa_{\star}=\frac{1}{1+\frac{1}{2} c_{14}} \kappa^{\circledast}=\frac{1-\frac{1}{2} \alpha}{1+\frac{1}{2} c_{14}} \kappa_{\infty}^{Æ}$, so that whenever we need $\kappa_{\infty}$ we could relate it to the textbookvalue $\kappa_{\star}$ by $\kappa_{\infty}=\kappa_{\star}$ (in the GR case), or $\kappa_{\infty}=\frac{1+\frac{1}{2} c_{14}}{1-\frac{1}{2} \alpha} \kappa_{\star}$ (in the $Æ$-case). Obviously, when $c_{14}=-\alpha, \kappa_{\infty}^{Æ}=\kappa_{\infty}^{\mathrm{GR}}$, the background cosmologies are the same in GR and our Æ -model.

[21] J.D. Barrow and R.J. Scherrer, Phys. Rev. D 70, 103515 (2004); T. Clifton, J.D. Barrow and R.J. Scherrer, Phys. Rev. D 71, 123526 (2005).

[22] T. Clifton, D. F. Mota and J. D. Barrow, Mon. Not. Roy. Astron. Soc. 358 (2005) 601 arXiv:gr-qc/0406001.

[23] C. M. Will, Theory and Experiment in Gravitational Physics (Cambridge University Press, Cambridge, 1993).

[24] See, for example, A. R. Liddle and D. H. Lyth, Cosmological Inflation and Large-Scale Structure (Cambridge University Press, Cambridge, 2000).

[25] T. Koivisto and D. F. Mota, Phys. Rev. D 73, 083502 (2006) arXiv:astro-ph/0512135.

[26] T. Koivisto and D. F. Mota, arXiv:0707.0279 [astro-ph].

[27] D. F. Mota, J. R. Kristiansen, T. Koivisto and N. E. Groeneboom, arXiv:0708.0830 [astro-ph].

[28] With general choices of parameters $c_{1,2,3,4}$, Eq. (71) is only valid if $\mathcal{H}^{\prime}-\mathcal{H}^{2} \simeq 0$. Thus, in the radiationdominated era it cannot apply even for superhorizon scales, and its solution Eq. (72) should be modified as well.

[29] T. G. Zlosnik, P. G. Ferreira and G. D. Starkman(2007), arXiv: 0711.0520 [astro-ph].

[30] E. A. Lim, private communication.

[31] H. Zhao (2007), Astrophys. J. Lett., in press. (arXiv: 0710.3616 [astro-ph]).

[32] A. M. Lewis, A. Challinor and A. Lasenby, Astrophys. J. 538, 473 (2000). See also http://camb.info/ 